\title{
Efecto de la frecuencia de riego en parámetros cuantitativos y cualitativos de la Vitis vinifera cv. Syrah
}

\author{
Montoro Rodríguez, A. ${ }^{1}$, Picazo Denia ${ }^{1}$, H., Mañas Jiménez, F. ${ }^{1}$
}

1 Instituto Técnico Agronómico Provincial; meli.itap@dipualba.es

Resumen: La implantación generalizada de riego por goteo en cultivos leñosos nos ha motivado al estudio de la frecuencia del riego en dichos cultivos, ya que por definición el riego por goteo se ha considerado un riego de "bajo caudal y alta frecuencia". Los cultivos leñosos presentan unas características morfológicas y estructurales muy diferentes a los cultivos herbáceos, para los que se diseñó en su origen el riego por goteo, entre los que se encuentra su sistema radicular más extenso y profundo, con una capacidad mayor de exploración del terreno. Además de ello, estudios previos [1], indican pérdidas de agua por evaporación en cada evento de riego, por lo que a priori, modificar el manejo del riego por goteo en este tipo de cultivos, reduciendo la frecuencia de riego e incrementando el número de horas del mismo, parece razonable. Es por ello que abordamos en Castilla-La Mancha, región con clima semiárido y con cerca de 160.000 ha de viñedo para vinificación en regadío [2], el estudio que se presenta, ya que pensamos que se puede mejorar la eficiencia del agua de riego en la vid, simplemente con el manejo del mismo. El ensayo se realizó en Valdeganga (Albacete) en un cultivar de la variedad Syrah, conducido en espaldera a un marco de plantación de $3 \times 1.25 \mathrm{~m}$., suelo franco arcillo arenoso y sin limitaciones de profundidad. El planteamiento del ensayo se basó en un diseño de bloques al azar cuyo factor fue la "frecuencia de riego": I1) Un riego semanal; I2) dos riegos semanales. Las programaciones de riego se ajustaron para unas necesidades hídricas de un tercio de la ETo, con balance de agua en el suelo. Durante todo el ciclo vegetativo del cultivo se ha medido semanalmente el estrés hídrico, a través de los potenciales de hoja y tallo, así como medidas de intercambio gaseoso con la finalidad de parametrizar al máximo el cultivo y poder detectar diferencias en el comportamiento fisiológico de ambos tratamientos. En cuanto al suelo, se ha medido de forma continua el contenido de agua en el mismo con sondas Sentek Drill \& Drop, a 6 profundidades diferentes (5, 15, 25, 35, 45, $55 \mathrm{~cm})$. En vendimia se analizó la producción, así como la madurez tecnológica y fenólica de la uva. Los resultados, tras un año de experimentación, nos muestran si es posible un manejo diferente, al que se está realizando actualmente, del riego por goteo en el cultivo de la vid.

Palabras clave: Vitis vinifera; frecuencia de riego; producción. 


\title{
Effect of irrigation frequency on quantitative and qualitative parameters of Vitis vinifera cv. Syrah
}

\author{
Montoro Rodríguez, A. ${ }^{1}$, Picazo Denia ${ }^{1}$, H., Mañas Jiménez, F. ${ }^{1}$
}

1 Instituto Técnico Agronómico Provincial; meli.itap@dipualba.es

\begin{abstract}
The widespread implementation of drip irrigation in woody crops has motivated us to study the frequency of irrigation in these crops since by definition drip irrigation has been considered a "low flow and high frequency" irrigation. Woody crops have morphological and structural characteristics that are very different from herbaceous crops, for which drip irrigation was originally designed, among which is their more extensive and deeper root system, with a greater capacity for exploring the terrain. In addition to this, previous studies [1], indicate losses of water by evaporation in each irrigation event, so a priori, modify the management of drip irrigation in this type of crops, reducing the frequency of irrigation and increasing the number of hours of it, it seems reasonable. That is why we approached in Castilla-La Mancha, a region with a semi-arid climate and with about 160,000 ha of vineyards for irrigated winemaking [2], the study that is presented, since we think that efficiency can be improved of irrigation water on the vine, simply by managing it. The test was carried out in Valdeganga (Albacete) on a cultivar of the Syrah variety, conducted on a trellis to a plantation frame of $3 \times 1.25 \mathrm{~m}$., Sandy clay loam soil and without depth limitations. The trial approach was based on a randomized block design whose factor was the "irrigation frequency": I1) One weekly irrigation; I2) two weekly irrigations. The irrigation schedules were adjusted for the water needs of one-third of the ETo, with a water balance in the soil. During the entire vegetative cycle of the crop, the water stress has been measured weekly, through the potential of the leaf and stem, as well as gas exchange measures to parameterize the crop as much as possible and to be able to detect differences in the physiological behavior. of both treatments. Regarding the soil, the water content in it has been continuously measured with Sentek Drill \& Drop probes, at 6 different depths $(5,15,25,35,45,55 \mathrm{~cm})$. During the harvest, the production was analyzed, as well as the technological and phenolic maturity of the grape. The results, after a year of experimentation, show us if different management is possible, from the one currently being carried out, of drip irrigation in vine cultivation.
\end{abstract}

Keywords: Vitis vinifera; irrigation frequency; production 


\section{Congreso Nacional de Riegos CARTAGENA 2021}

\section{Introducción}

Por definición el riego por goteo se considera un riego de "bajo caudal y alta frecuencia", localizado cerca del sistema radicular. Desde su origen primitivo en China, durante el siglo I a. de C., con el uso de vasijas de barro enterradas y sin esmaltar, hasta cómo lo conocemos en la actualidad, ha sufrido modificaciones técnicas en cuanto a materiales y forma de aplicar "la gota de agua" en el terreno. En la década de 1920, los productores alemanes comenzaron a utilizar tuberías perforadas para regar sus plantaciones. Una vez que los plásticos se desarrollaron y se usaron ampliamente después de la Segunda Guerra Mundial, un inventor australiano llamado Hannis Thill comenzó a usar una configuración de tubería de plástico específica con pasillos largos para distribuir el agua de riego de manera uniforme a los cultivos. En 1959, Simcha Blass y Kibbutz Hatzerim desarrollaron y patentaron el primer emisor de riego por goteo. El concepto de emisor fue desarrollado varios años antes por Simcha y su hijo Yeshayahu en Israel. En lugar de liberar agua a través de pequeños orificios bloqueados fácilmente por partículas diminutas, el agua se liberó a través de pasillos cada vez más largos, lo que disminuyó la velocidad del agua a medida que salía de la tubería [3]. A lo largo de su evolución y desarrollo, se ha pretendido siempre mejorar el sistema desde el punto de vista técnico, pero poco se ha trabajado sobre la adaptación del sistema de riego y su manejo en cada uno de los cultivos regados. Actualmente se riegan con este sistema cultivos tan diferentes como hortícolas, con un sistema radicular fasciculado, corto y poco ramificado, como por ejemplo la cebolla, hasta cultivos leñosos como por ejemplo el nogal, en el que las raíces primarias crecen como un tronco desde la base del árbol y hacia las profundidades del suelo y tienen raíces secundarias que crecen de ellas en todas direcciones hasta unos $45 \mathrm{~cm}$ del suelo, extendiéndose en distancia de dos a tres veces el diámetro de la copa del árbol. El caso que nos ocupa es el de la vid, con un sistema radicular diferente a los descritos y que podríamos catalogar como "intermedio" entre ambos, ya que se trata de un sistema radical adventicio, fasciculado y ramificado. La extensión de sistema radicular depende de la especie, marco de plantación, tipo de suelo y técnicas de cultivo, pero como generalidad podríamos decir que el $90 \%$ del sistema radical se desarrolla por encima del primer metro de suelo, estando la gran mayoría entre los 40 y $60 \mathrm{~cm}$ de profundidad.

El impacto de la dosis de riego en el cultivo de la vid (Vitis vinifera L.) y su distribución en diferentes fases del ciclo sobre los principales aspectos agronómicos y enológicos se han estudiado en profundidad [4-8]. Sin embargo, los efectos de la frecuencia de riego apenas se han estudiado en este cultivo, aunque se ha demostrado que tiene un impacto relevante en otras especies como manzano [9] y olivo [10]. La frecuencia de riego afecta la distribución espacial y temporal del agua en el suelo, ya que depende de las características hidráulicas del perfil [11]. En suelos ligeros, se han observado aumentos de rendimiento atribuidos a aportaciones de riego con frecuencias altas [12] mientras que en suelos arcillosos este efecto se ha relacionado con frecuencias de riego más bajas [13-14]. En condiciones de clima cálido y suelos de textura media (franco arcillo arenosos), las altas frecuencias de riego aumentan las pérdidas de agua debido a la evaporación en las zonas más superficiales capas del suelo (Montoro et al.2016) [1], aumentando el déficit hídrico cuando se dispone de concesiones bajas de agua.

El objetivo de este trabajo es estudiar el efecto de la frecuencia de riego en parámetros cuantitativos y cualitativos de la Vitis vinifera cv. Syrah en un viñedo cultivado en clima semiárido en Castilla-La Mancha. 


\section{Congreso Nacional de Riegos CARTAGENA 2021}

\section{Materiales y métodos (Trabajo de innovación: descripción de la innovación)}

La parcela en la que se ha llevado a cabo el ensayo está situada en la localidad de Valdeganga (Albacete), a $667 \mathrm{msm}$. La superficie total es de 10 ha de Vitis vinifera cv. Syrah injertada sobre 110 Richter y conducida en espaldera con sistema de poda Guyot. En el viñedo se acotó una zona de 0.8 ha sobre la que se realizó el estudio. La plantación tiene una edad de 16 años, a un marco de $3 \mathrm{~m}$ entre líneas de cultivo y 1,25 m entre cepas de la misma línea y regada desde el primer año de cultivo. La clasificación del suelo según FAO (1988) es Calcisol cámbico, con textura franco arenosa y sin limitaciones de profundidad para el desarrollo radicular. El clima es semiárido, con una precipitación media anual de los últimos 10 años de $356 \mathrm{~mm}$. Las condiciones climáticas de humedad y temperatura del aire, así como precipitación, fueron medidas durante el experimento con una estación agroclimática FieldClimate ${ }^{\circledR}$ localizada en la misma parcela del ensayo.

En 2021 se diseñó un ensayo experimental de bloques al azar con 3 repeticiones y 2 tratamientos: un riego por semana (R1) y dos riegos por semana (R2). Se aportó en ambos casos y con un balance semanal, un 30\% de la evapotranspiración de referencia según Penman-Monteith [15] hasta vendimia, mediante riego localizado por goteo, con dos goteros autocompensantes por planta, el R1 de caudal 4 1/h y el R2 de $81 / h$.

El estado hídrico de las plantas se realizó mediante medidas de potencial hídrico de hoja ( $\Psi 1)$ y potencial hídrico de tallo ( $\Psi$ s ) a medio día solar, al tercer día de riego en ambos tratamientos. Para ello se utilizó una cámara de presión tipo Scholander (PMS Instruments Company, Albany, Oregón, USA). El índice de área foliar se obtuvo con la relación alométrica de medida de longitud de pámpano y área foliar, obtenida el mismo año para el viñedo en estudio. Las medidas de intercambio gaseoso se realizaron con un equipo Li-Cor 6400 XT. La humedad del suelo se midió con sondas Drill\&Drop ${ }^{\circledR}$ a cinco profundidades $5,15,25,35,45$ y $55 \mathrm{~cm}$. Cuando las bayas llegaron a $23^{\circ}$ Brix de solidos solubles, se realizó la vendimia, obteniéndose los componentes del rendimiento $\left(\mathrm{n}^{\mathrm{o}}\right.$ de racimos, bayas por racimo y peso de la baya) y se analizó la madurez tecnológica y fenólica $(\mathrm{pH}$, ácido málico, ácido tartárico, ácido D-glucónico, acidez total, antocianos, índice de polifenoles totales, intesidad de color, nitrógeno fácilmente asimilable y potasio). Los resultados obtenidos se analizaron estadísticamente mediante análisis de la varianza utilizando el programa estadístico IBM SPSS v.19®.

\section{Resultados y discusión}

La Tabla 1 muestra los valores de ET0 calculados por Penman-Monteith 56 [15], precipitación, riego y agua total recibida en los tratamientos ensayados a lo largo de todo el ciclo vegetativo del cultivo, habiéndose cubierto con agua de lluvia las necesidades hídricas desde brotación hasta julio, mes en el que se iniciaron los riegos, en un estado fenológico entre baya tamaño guisante y el cierre del racimo, como se muestra en la tabla 2 , en la que se puede ver que la fecha de alcanzar los estados fenológicos fue la misma para los dos tratamientos hasta finales de julio, en el que se inició el envero antes para el tratamiento $\mathrm{R} 2$ o el de dos riegos semanales.

A pesar de haber tenido una misma cantidad de agua total recibida en los dos tratamientos, se ha apreciado un diferente comportamiento en el estado hídrico entre ambos. Así, el tratamiento R1 o de un riego semanal, se mostró ligeramente con un potencial menor que el R2 o tratamiento de dos riegos semananles, como puede observarse en la figura 1, en la que se refleja los potenciales de tallo medidos a medio día solar durante el ensayo, marcándose la diferencia entre los dos tratamientos desde el primer riego. 


\section{Congreso Nacional de Riegos CARTAGENA 2021}

Tabla 1. Demanda evaporativa de la atmósfera, precipitación, riego y agua total recibida en los tratamientos.

\begin{tabular}{lcrrr}
\hline Meses & $\begin{array}{c}\text { ET0 } \\
(\mathbf{P M})\end{array}$ & $\begin{array}{c}\text { Pe } \\
(\mathbf{m m})\end{array}$ & $\begin{array}{c}\text { Riego } \\
(\mathbf{m m})\end{array}$ & $\begin{array}{c}\text { Agua total } \\
\text { recibida } \\
(\mathbf{m m})\end{array}$ \\
\hline Octubre & 80,60 & 29,1 & 0 & 29,1 \\
\hline Noviembre & 35,10 & 67,9 & 0 & 67,9 \\
\hline Diciembre & 31,00 & 24,1 & 0 & 24,1 \\
\hline Enero & 28,70 & 61,7 & 0 & 61,7 \\
\hline Febrero & 52,70 & 16,4 & 0 & 16,4 \\
\hline Marzo & 70,50 & 16,1 & 0 & 16,1 \\
\hline Total parada vegetativa & $\mathbf{2 9 8 , 6 0}$ & $\mathbf{2 1 5 , 3}$ & $\mathbf{0}$ & $\mathbf{2 1 5 , 3}$ \\
\hline Abril & 85,40 & 65,0 & 0 & 65,0 \\
\hline Mayo & 157,10 & 33,2 & 0 & 33,2 \\
\hline Junio & 181,10 & 62,0 & 0 & 62,0 \\
\hline Julio & 231,60 & 0 & 42,70 & 42,70 \\
\hline Agosto & 196,60 & 33,4 & 85,40 & 118,8 \\
\hline Septiembre & 60,20 & $41,2\left(^{*}\right)$ & 0 & 41,2 \\
\hline Total brotación-vendimia & $\mathbf{9 1 2 , 0 0}$ & $\mathbf{1 2 8 , 6}$ & $\mathbf{1 2 8 , 0}$ & $\mathbf{3 6 2 , 9}$ \\
\hline Total & $\mathbf{1 2 1 0 , 6 0}$ & $\mathbf{4 5 0 , 3}$ & $\mathbf{1 2 8 , 0}$ & $\mathbf{5 7 8 , 2}$ \\
\hline
\end{tabular}

${ }^{*}$ ) Lluvia caída después de vendimia

Tabla 2. Fecha de Estado fenológico en los tratamientos

\begin{tabular}{l|l|l}
\hline Fecha & Tratamiento R1 & Tratamiento R2 \\
\hline $15 / 04 / 2021$ & Brotación & Brotación \\
\hline $03 / 05 / 2021$ & Hojas extendidas & Hojas extendidas \\
\hline $10 / 06 / 2021$ & Floración & Floración \\
\hline $16 / 06 / 2021$ & Cuajado & Cuajado \\
\hline $28 / 06 / 2021$ & Baya tamaño guisante & Baya tamaño guisante \\
\hline $19 / 07 / 2021$ & Cerramiento del racimo & Inicio envero \\
\hline $26 / 07 / 2021$ & Inicio de envero & Envero 20\% \\
\hline $02 / 08 / 2021$ & Envero 50\% & Envero 75\% \\
\hline $09 / 08 / 2021$ & Envero $75 \%$ & $100 \%$ enveradas \\
\hline $16 / 08 / 2021$ & $100 \%$ enveradas & Maduración \\
\hline $26 / 08 / 2021$ & Vendimia & Vendimia \\
\hline
\end{tabular}

La figura 2 muestra los valores de fotosíntesis en los tratamientos. A partir de finales de julio se aprecia una fotosíntesis ligeramente mayor en el tratamiento de dos riegos semanales, aunque no se han encontrado diferencias significativas entre tratamientos en el análisis estadístico.

El contenido de humedad del suelo se refleja en la figura 3, en la que se representa el valor medio diario en cada uno de los tratamientos, a la profundidad de $5,15,25,35,45$ y $55 \mathrm{~cm}$ y la suma total de todas ellas en el perfil. Los picos en las gráficas indican los riegos aportados. La media y resumen de todas ellas se presenta en la figura 4, en la que se observa una humedad en todo el perfil del suelo mayor en el tratamiento R1 que en el R2, la explicación que encontramos a ello puede ser debido al efecto de evaporación, que debe ser mayor en tratamientos de 2 riegos semanales que en tratamientos de 1 riego semanal (Montoro et al., 2016) [1]. 


\section{CDERY \\ XXXVIII Congreso Nacional de Riegos CARTAGENA 2021}

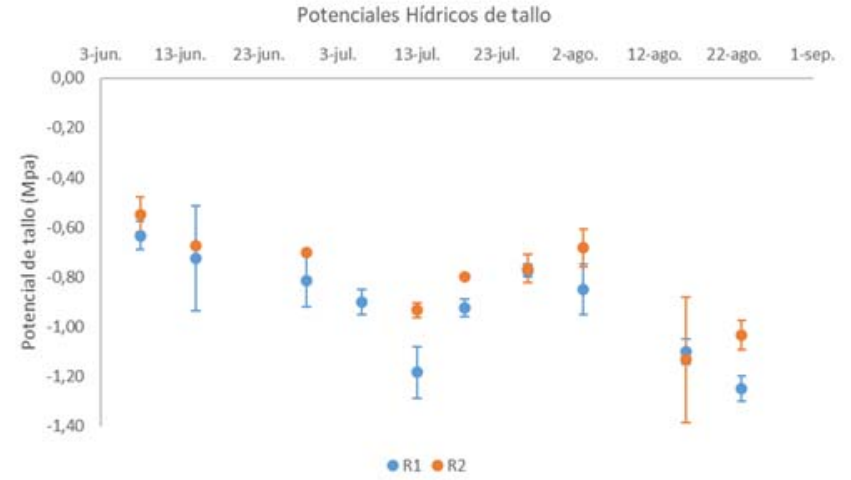

Figura 1. Potenciales hídricos de tallo tomados a medio día solar.

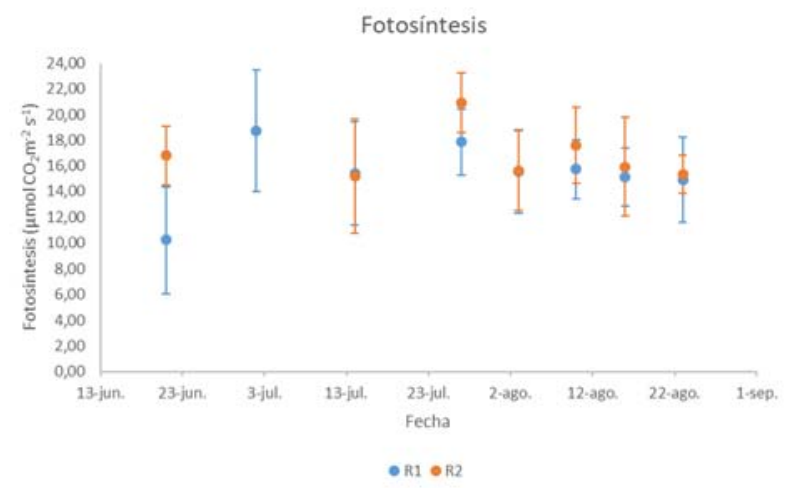

Figura 2. Fotosíntesis en los tratamientos estudiados.
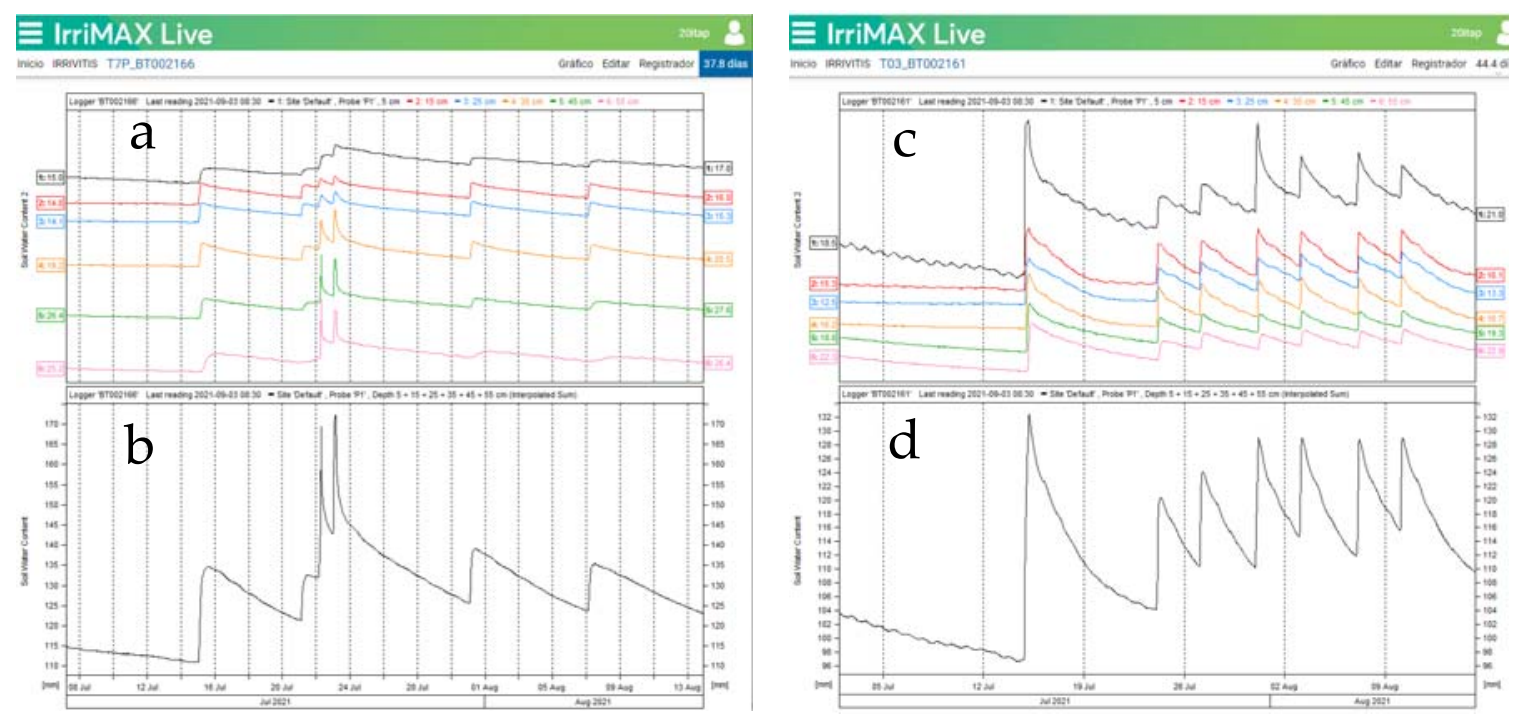

Figura 3. Contenido de humedad en el suelo en el tratamiento R1 a 6 profundidades (a) y total (b) y en el tratamiento R2 a 6 profundidades (c) y total (d).

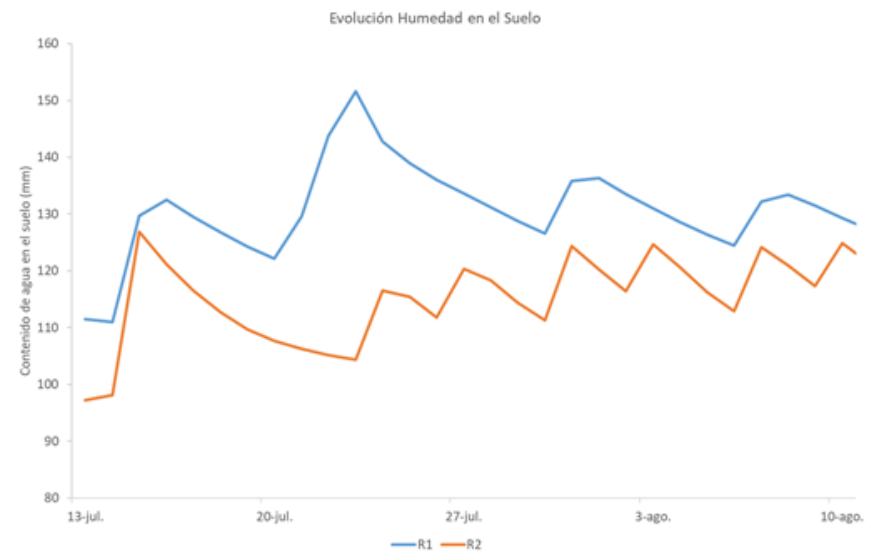

Figura 4. Comparativa del contenido total de humedad en el suelo en ambos tratamientos 


\section{Congreso Nacional de Riegos CARTAGENA 2021}

A pesar de que la fecha de vendimia estaba programada para la recolección con $23^{\circ}$ Brix, se tuvo que realizar la de los dos tratamientos a la vez, por motivos de previsión de granizo, lluvias y fuertes vientos huracanados. Se realizó el 26 de agosto, fecha en la que ninguno de los tratamientos había alcanzado los $23^{\circ}$ Brix. El R1 se encontraba en $22,7^{\circ}$ Brix y el R2 en 20,5 $5^{\circ}$ Brix, como se observa en la figura 5 , por lo que vemos que la frecuencia de riego sí que afectó a la fecha de maduración de la uva, siendo más rápida en los tratamientos de un riego semanal, que en los de 2 riegos semanales, aunque sin significación estadística. En cuanto al rendimiento, la figura 6, muestra prácticamente el mismo rendimiento en ambos tratamientos, 2,04 vs 2,16 t/ha, en R1 y R2, respectivamente.

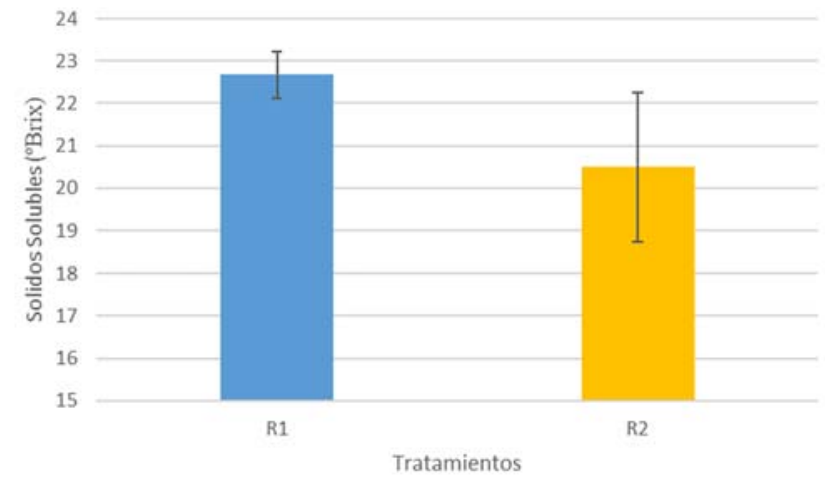

Figura 5. ${ }^{\circ}$ Brix en los dos tratamientos a misma fecha (finales de agosto).

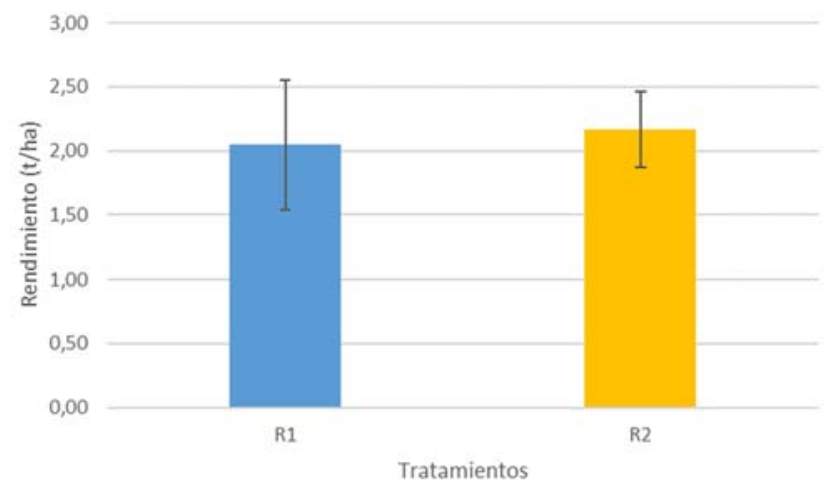

Figura 6. Rendimiento en los dos tratamientos a misma fecha (finales de agosto).

En cuanto a la acidez, los dos tratamientos tuvieron similar ácido tartárico $(5,4 \mathrm{~g} / \mathrm{l})$, pero sí que hubo una diferencia en el ácido málico ( 2,4 vs 3,1 g/l en R1 vs R2), debido quizá al diferente estado de maduración de la uva, ello produjo una acidez total también diferente, siendo mayor en el tratamiento R2 (6,4 g/l) que en el R1 (5,8 g/l). El pH fue el mismo en ambos casos, alcanzando un valor de 3,4.

Referente a la maduración fenólica, las figuras 7 y 8 muestran el valor de los antocianos e índice de polifenoles totales, observándose diferencia entre los dos tratamientos, siendo mayores los valores en ambos casos para el tratamiento de un riego semanal (R1). Esto produjo también una intensidad de color mayor en R1 que en R2 (3,58 vs 1,51).

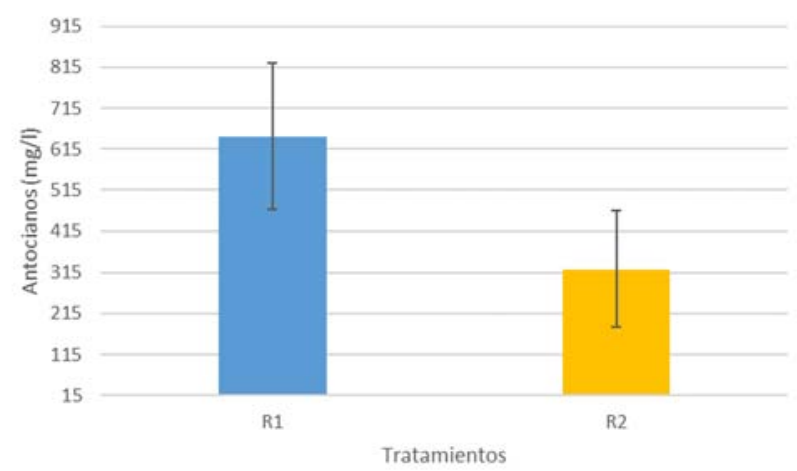

Figura 7. Antocianos en los dos tratamientos a misma fecha (finales de agosto).

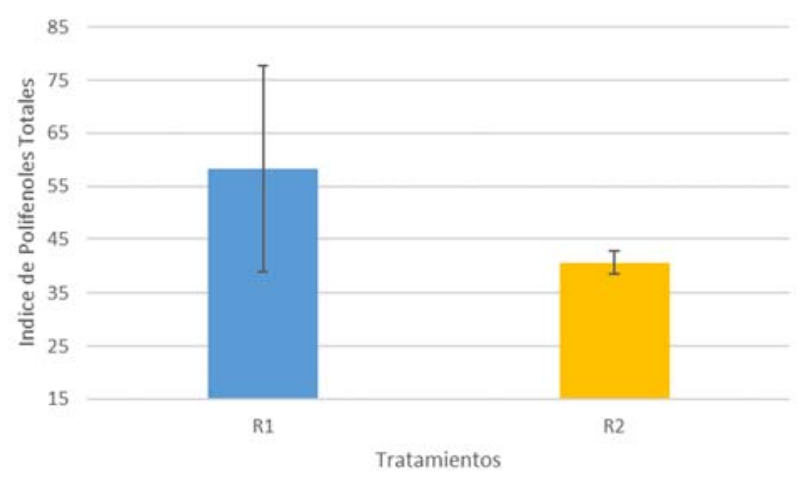

Figura 8. Índice de polifenoles totales en los dos tratamientos a misma fecha (finales de agosto). 


\section{Congreso Nacional de Riegos CARTAGENA 2021}

El nitrógeno fácilmente asimilable por las levaduras, también alcanzó valores diferentes, siendo mayor en el tratamiento de 2 riegos semanales (R2), como muestra la figura 9, aunque las diferencias encontradas no fueron significativas.

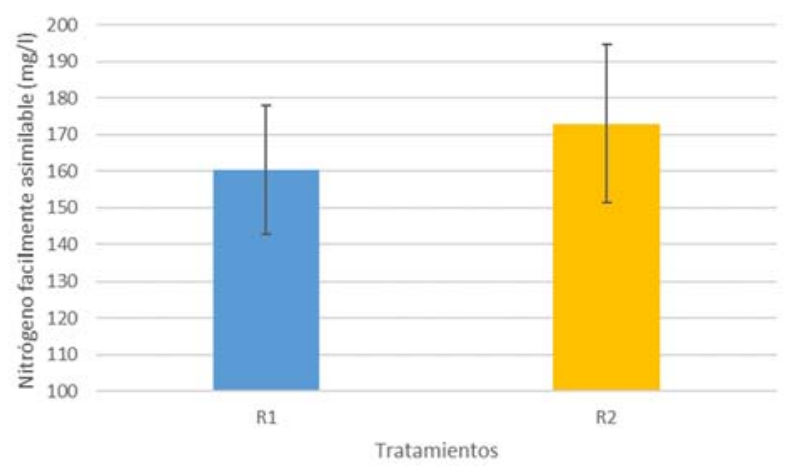

Figura 9. Nitrógeno fácilmente asimilable en los dos tratamientos a misma fecha (finales de agosto).

\section{Conclusiones}

De un solo año de estudio no se pueden obtener conclusiones firmes, pero los resultados parecen indicar que la frecuencia de riego depende del tipo de cultivo a regar, ya que, como se ha visto, en un cultivo como vid, se han obtenido buenos resultados, e incluso similares con un sólo riego semanal que con dos riegos semanales, algo impensable en cultivos hortícolas, como por ejemplo, ajo o cebolla.

La frecuencia de riego parece ser que afecta a la rapidez de la maduración, así, en nuestro caso y en el cultivo de la vid, la maduración se ha alcanzado antes en el tratamiento de un solo riego que en el de dos riegos semanales.

El rendimiento y maduración tecnológica, no se han visto afectados por las dos frecuencias de riego ensayadas (uno y dos riegos semanales). En cambio, sí que se han encontrado diferencias en la maduración fenólica entre las dos frecuencias de riego, alcanzando valores mayores en el tratamiento de un riego semanal, aunque esto ha podido estar motivado por el diferente grado de maduración de la uva.

Se requiere de los resultados de los próximos años para validar las primeras conclusiones obtenidas en este estudio.

\section{Agradecimientos}

Los autores agradecen la financiación obtenida del Ministerio de Ciencia e Innovación, a través de las ayudas a Proyectos I+D+i , en el marco de los Programas Estatales de Generación de Conocimiento y Fortalecimiento Científico y Tecnológico del Sistema I+D+i y de I+D+i orientada a los Retos de la Sociedad, al proyecto PID2019-105039RR-C43.

\section{Referencias}

1. Montoro, A.; Mañas, F.; López-Urrea., R. Transpiration and evaporation of grapevine, two components related to irrigation strategy. Agricultural Water Management. 2016. Volume 177, November 2016, Pages 193-200 . https://doi.org/10.1016/j.agwat.2016.07.005

2. MAPYA. Avance del Anuario de Estadística Agraria. 2020. https://www.mapa.gob.es/es/estadistica/temas/publicaciones/anuario-de-estadistica/default.aspx 


\section{Congreso Nacional de Riegos CARTAGENA 2021}

3. Dripdepot. The History of Drip Irrigation. https://help.dripdepot.com/support/solutions/articles/11000044434-the-historyof-drip-irrigation. 2017.

4. Kliewer, W.M.; Freeman, B.M.; Hosssom, C. Effect of Irrigation, Crop Level and Potassium Fertilization on Carignane Vines. I. Degree of Water Stress and Effect on Growth and Yield. Am J Enol Vitic. 1983. Vol 34: 186-196.

5. Poni, S.; Lakso, A.N.; Turner, J.R.; Melrous, R.E. The effects of pre- and post-veraison water stress on growth and physiology of potted Pinot Noir grapevines at varying crop levels. Vitis, 1993. Vol 32, 207-2014.

6. Ojeda, H.; Andary, C.; Kraeva, E.; Carbonneau, A.; Deloire, A. Influence of Pre- and Postveraison Water Deficit on Synthesis and Concentration of Skin Phenolic Compounds during Berry Growth of Vitis vinifera cv. Shiraz. Am J Enol Vitic., 2002. Vol. 53: 261-267.

7. Intrigliolo, D.S; Castel, J.R. Response of grapevine cv. Tempranillo to timing and amount of irrigation: water relations, vine growth, yield and berry and wine composition. Irrig Sci 2010. Vol. 28, 113-125.

8. Santesteban, L.G.; Miranda, C.; Royo, J.B. Regulated deficit irrigation effects on growth, yield, grape quality and individual anthocyanin composition in Vitisvinifera L. cv. 'Tempranillo'. Agric Water Manag, 2011. Vol 98, 1171-1179.

9. Levin, I.; Assaf, R; Bravdo, B. 1979. Irrigation, water status and nutrient uptake in an apple orchard. ISHS Acta Hort , 1979, Vol 92

10. Palomo, M.J.; Moreno, F.; Fernández, J.E.; Díaz-Espejo, A.; Girón, I.F. Determining water consumption in olive orchards using the water balance approach. Agric Water Manag, 2002. Vol 55(1), 15-35.

11. Hung, J.Y.T. Determination of emitter spacing and irrigation run time including plant root depth. In: Lamm FR (ed) Microirrigation for a changing world: conserving resources/ preserving the environment. Proceedings of the fifth international microirrigation congress, Orlando, Florida, 2-6 April, 1995. ASAE, St. Joseph, Mich., pp 292-296.

12. Myburgh, P.A. Comparing Irrigation Systems and Strategies for Table Grapes in the Weathered Granite-gneiss Soils of the Lower Orange River Region. S. Afr. J. Enol. Vitic.. 2012. Vol 33 (2), 184-197.

13. Selles G.; Ferreyra, E.; Contreras, R.E.; Ahumada, G.W.; Valenzuela, J; Bravo, R.V. Effect of three irrigation frequencies applied by drip irrigation over table grapes (Vitis vinifera L. cv. Thompson Seedless) located in the Aconcagua Valley (Chile). ISHS Acta Horticulturae. 2004. Vol 646

14. Sebastian, B.; Lissarrague, J.R.; Santesteban, L.G., et al. Effect of irrigation frequency and water distribution pattern on leaf gas exchange of cv.' Syrah ' grown on a clay soil at two levels of water availability. Agric Water Manag. 2016. Vol. 177:410418. doi: 10.1016/j.agwat.2016.08.032

15. Allen, R.G.; Pereira, L.S.; Raes, D.; Smith, M. Crop evapotranspiration. Guidelines for computing crop water requirements. FAO Irrigation and Drainage. 1998. Paper no. 56, FAO, Rome. 